PAPER

\title{
Design of an ultrasonic piezoelectric transducer having double-linked diaphragms for parametric speakers
}

\author{
Jun Kuroda ${ }^{1, *}$, Yasuhiro Oikawa ${ }^{1}$, Yoshio Yamasaki ${ }^{2}$, Shigeo Sato ${ }^{3}$, \\ Motoyoshi Komoda ${ }^{3}$ and Yasuharu Onishi ${ }^{3}$ \\ ${ }^{1}$ Department of Intermedia Art and Science, Waseda University, \\ 3-4-1 Ohkubo, Shinjuku-ku, Tokyo, 169-8555 Japan \\ ${ }^{2}$ Waseda University, \\ 3-4-1 Ohkubo, Shinjuku-ku, Tokyo, 169-8555 Japan \\ ${ }^{3}$ NEC Corporation, \\ 5-7-1, Shiba, Minato-ku, Tokyo, 108-8001 Japan
}

(Received 11 June 2014, Accepted for publication 25 March 2015)

\begin{abstract}
Small highly efficient ultrasonic transducers are in high demand to fabricate compact parametric speakers. We focus on small piezoelectric transducers that can be used for ultrasonic emitters of parametric speakers. Typical small piezoelectric transducers have piezoelectric boards and radial cones. We propose a transducer consisting of a unimorph diaphragm and a flat metal plate. This structure gives two resonant peaks, which are determined by the physical parameters and dimensions of the diaphragms, the flat plate, and their junction structure. Controlling these resonant peaks is one approach to fabricating transducers having the ideal frequency response of the sound pressure for parametric speakers. We devised a design to control these two resonant peaks by adjusting the junction structure of the diaphragm and the flat plate. Using the results of a theoretical analysis, we designed an improved transducer that satisfies the ideal frequency response for parametric speakers.
\end{abstract}

Keywords: Piezoelectric transducer, Ultrasonic, Parametric speaker

PACS number: 43.38.Fx, 43.40.At [doi:10.1250/ast.36.385]

\section{INTRODUCTION}

Parametric speaker arrays have been studied for speech privacy systems since Westervelt presented his paper on parametric acoustic arrays (1963) [1-4]. Mainly parametric speakers have been studied from the point of view of sound signal processing, arrangement of transducers, and nonlinear acoustical phenomena. Seminal studies have established the technologies relating to the structure of piezoelectric transducers; however, there is a dearth of information on fabricating piezoelectric transducers to produce parametric speakers having high-quality sound [5,6]. In this paper, we focus on small piezoelectric transducers that can be used for parametric speakers.

Input signals to parametric speakers consist of audible sound modulated by a carrier wave, which has a bandwidth with an audible range of about $20 \mathrm{kHz}$. Sound demodulated in air according to self-demodulation of the amplitude modulation (AM) has a frequency response proportional to the square of the frequency. Therefore, the use of ultrasonic

*e-mail: jun-kuroda@suou.waseda.jp transducers is required for the frequency response of the sound pressure level to decrease by $12 \mathrm{~dB}$ per octave from the resonant frequency. One approach to satisfying this requirement is to efficiently use the resonant peaks of vibration of the transducers. Namely, if a dominant parameter and governing equations are specified, the resonant peaks of vibration can be accurately designed, achieving the required frequency responses of the transducers. To study this issue, we employ transducers that consist of double-linked flat disks.

The dominant parameter and the governing equations were derived from mechanical properties, i.e., the dimensions and the junction structure of diaphragms and a rod.

\section{THEORETICAL ANALYSIS}

\subsection{Ultrasonic Transducer}

Conventional ultrasonic piezoelectric transducers using bending vibration have the structure depicted in Fig. 1. This type of transducer has a radial cone and a diaphragm, both of which are made of metal. The radial cone and the diaphragm are fixed by a resin glue. The transducer shown in Fig. 1 has two resonant peaks, which are caused by the 


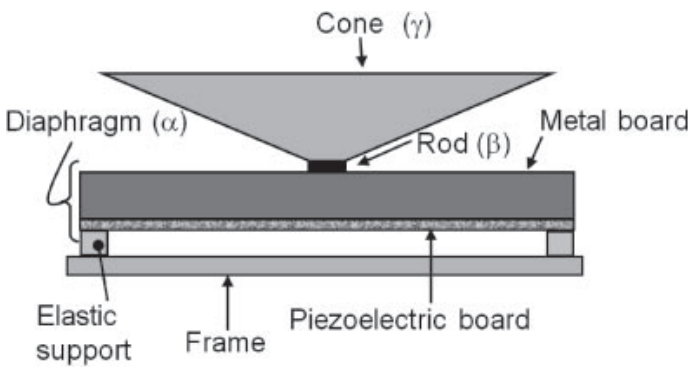

Fig. 1 Schematic of conventional ultrasonic piezoelectric transducer.

flap vibration mode of the radial cone and the diaphragm [7]. The resonant frequencies and the vibration amplitudes of two resonant peaks are determined by the mechanical properties and dimensions of the diaphragms and their junction structure.

The sound pressure level of a self-demodulated sound of an AM sine wave is derived from the Westervelt equation as [1-3]

$$
p_{s}(t)=-\frac{\beta p_{0}^{2} a^{2} m \omega_{s}^{2}}{8 \rho_{0} c_{0}^{4} \alpha_{c} r} \sin \omega_{s}\left(t-\frac{r}{c_{0}}\right),
$$

where $\beta$ is the parameter of nonlinearity, $\omega_{s}$ is the angular frequency of the signal, $\rho_{0}$ is the mass density of the air, $c_{0}$ is the speed of sound, $\alpha_{c}$ is the absorption coefficient of the carrier wave, $m$ is the degree of modulation, $p_{0}$ is the sound pressure level of the AM wave, $r$ is the distance from the transducer, and $a$ is the radius of the cross section of the primary sound beam.

Equation (1) shows that the sound pressure level of the demodulated sound is proportional to the square of the frequency of the demodulated sound. Therefore, when the sound pressure level of the transducers decreases by $12 \mathrm{~dB}$ per octave, that of the demodulated sound has a flat frequency response.

Therefore, in our design we expand the available bandwidth of the transducers by assignment two resonant frequencies while maintaining a high $Q$ factor. To assign resonant peaks close to each other, the mechanical properties of the diaphragms and the cone, i.e., the equivalent stiffness and mass of the transducers, must be closely matched. For this reason, we proposed an ultrasonic piezoelectric transducer having two flat diaphragms, as shown in Fig. 2 [8]. Diaphragm 1 is a unimorph-type piezoelectric transducer, which consists of an adhered metal board and a piezoelectric element. Diaphragm 2 consists of a metal board. A rod is used to link diaphragms 1 and 2 and should be made of an appropriately stiff resin to propagate the vibration to diaphragm 2 .

Systematic design of transducers requires clarifying the causes of resonance and antiresonance of the transducers. Therefore, it is important to formularize the relation

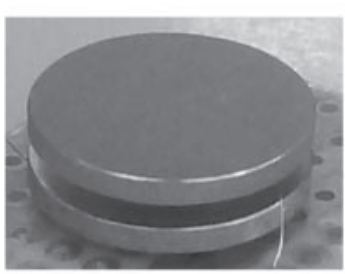

(a) Picture of transducer

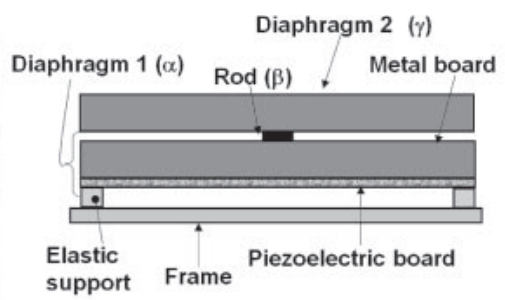

(b) Outline of transducer structure
Fig. 2 Schematic and photograph of proposed ultrasonic transducer and definitions of each part.

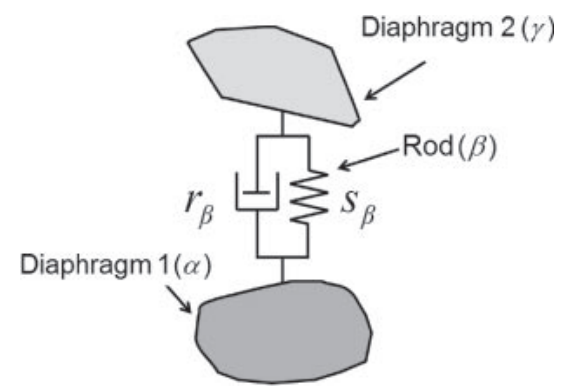

Fig. 3 Schematic of abstracted model of transducer.

between these resonant resonant frequencies and the mechanical properties of parts of the transducers. The equivalent circuit method is useful for analyzing and gaining an understanding of the behavior of the transducers.

\subsection{Derivation of the Equivalent Circuit for the Transducer}

There is considerable prior research on the acoustical equivalent circuit of piezoelectric transducers $[9,10]$. However, the detailed design of the resonant peaks of the transducer shown in Fig. 2 requires an equivalent circuit and calculation method to estimate the circuit constants from information on the structure, dimensions, and mechanical properties of the transducer and its parts. Therefore, we first derive the equivalent circuit of the mechanical element of the transducer from Lagrange's equation [11].

Figures 1 and 2 are abstracted as Fig. 3 for mathematical formulization. The rod is made of resin and the diaphragms are made of metal. Thus, the stiffness and mass density of the rod are much less than those of the diaphragms. Additionally, the total volume of the rod is much smaller than that of the diaphragms. Therefor, the mathematical analysis can be based on the following assumptions;

1. The mass of the rod is negligible. Hence the rod is represented by stiffness and viscous damping. The Lagrangian and dissipation function of the rod are 
determined by the difference of the averaged deflection at the junction area between the two diaphragms.

2. The mass density and the stiffness are uniform throughout diaphragms 1 and 2. However, viscous damping is affected by the junction area and glue.

From assumption 2, all metric tensors of the mass, the stiffness, and the viscous damping for arbitrary points in configuration space can be regarded as constants in the calculation process. The generalized coordinate of the $i$ th mass point includes all three rectangular coordinates $x^{i}, y^{i}$, $z^{i}$ without distinction. Hence, $3 N$ coordinates correspond to $N$ mass points.

By assumption 1, we consider only the general coordinates in diaphragms 1 and 2. Indices $\alpha$ and $\gamma$ denote those of the generalized coordinates to clarify the components of diaphragm 1 and diaphragm 2, respectively, i.e., diaphragm 1: $q^{\alpha(i)}$ and diaphragm 2: $q^{\gamma^{(i)}}$. Here, $\alpha(i)$ and $\gamma(i)$ are serial numbers.

When the number of the mass points in diaphragm 1 is $M, \alpha(i)$ and $\gamma(i)$ are $\alpha(i)=1,2, \ldots, 3 M$ and $\gamma(i)=3 M+1$, $3 M+2, \ldots, 3 N$, respectively. The Lagrangian, kinetic energy, potential, and dissipation function for each region are denoted by using a superscript, i.e., $L^{\alpha}, T^{\alpha}, U^{\alpha}$, and $J^{\alpha}$. Accordingly,

$$
L=L^{\alpha}+L^{\beta}+L^{\gamma} .
$$

Using the Einstein summation convention, the kinetic energy, the dissipation function, and the potential of the general coordinates $q^{i}$ and $q^{j}$ are

$$
\begin{aligned}
T=\frac{1}{2} m_{i j} d \dot{q}^{i} d \dot{q}^{j}, \quad J & =\frac{1}{2} r_{i j} d \dot{q}^{i} d \dot{q}^{j}, \\
\text { and } U & =\frac{1}{2} \kappa_{i j} d q^{i} d q^{j} .
\end{aligned}
$$

In the rectangular coordinate system, the formulas for $d q^{i}$ also hold for a generalized coordinate $q^{i}$; hence, all the above equations can be rewritten with the generalized coordinates.

Using the generalized coordinates $q^{\alpha(j)}$ and $q^{\gamma(j)}$, Lagrange's equations for diaphragms 1 and 2 are

$$
\begin{gathered}
\frac{d}{d t}\left(\frac{\partial T^{\alpha}}{\partial \dot{q}^{\alpha(j)}}\right)+\frac{\partial}{\partial q^{\alpha(j)}}\left(U^{\alpha}+U^{\beta}\right) \\
+\frac{\partial}{\partial \dot{q}^{\alpha(j)}}\left(J^{\alpha}+J^{\beta}\right)=f^{\alpha(i)}
\end{gathered}
$$

and

$$
\begin{aligned}
& \frac{d}{d t}\left(\frac{\partial T^{\gamma}}{\partial \dot{q}^{\gamma(j)}}\right)+\frac{\partial}{\partial q^{\gamma(j)}}\left(U^{\gamma}+U^{\beta}\right) \\
& \quad+\frac{\partial}{\partial \dot{q}^{\gamma(j)}}\left(J^{\gamma}+J^{\beta}\right)=f^{\gamma(i)}=0 .
\end{aligned}
$$

The Lagrangians of diaphragms 1 and 2 are represented as

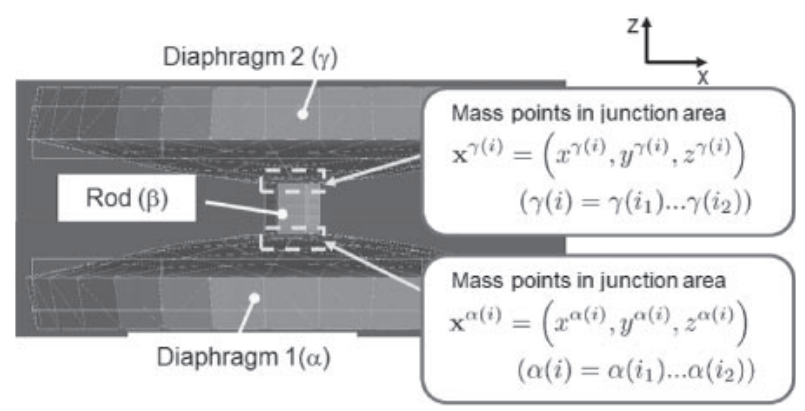

Fig. 4 Schematic of two diaphragms and junction area.

$$
L^{\alpha}=\frac{1}{2}\left\{m_{\alpha(i) \alpha(j)} \dot{q}^{\alpha(i)} \dot{q}^{\alpha(j)}-s_{\alpha(i) \alpha(j)} q^{\alpha(i)} q^{\alpha(j)}\right\}
$$

and

$$
L^{\gamma}=\frac{1}{2}\left\{m_{\gamma(i) \gamma(j)} \dot{q}^{\gamma(i)} \dot{q}^{\gamma(j)}-s_{\gamma(i) \gamma(j)} q^{\gamma(i)} q^{\gamma(j)}\right\}
$$

The dissipation functions are

$$
J^{\alpha}=\frac{1}{2} r_{\alpha(i) \alpha(j)} \dot{q}^{\alpha(i)} \dot{q}^{\alpha(j)}
$$

and

$$
J^{\gamma}=\frac{1}{2} r_{\gamma(i) \gamma(j)} \dot{q}^{\gamma(i)} \dot{q}^{\gamma(j)}
$$

As shown in Fig. 4, we define the generalized coordinates of the mass points in diaphragms 1 and 2, which belong to the junction area, as

$$
\boldsymbol{x}^{\alpha(i)}=\left(x^{\alpha(i)}, y^{\alpha(i)}, z^{\alpha(i)}\right), \quad \alpha(i)=\alpha\left(i_{1}\right) \cdots \alpha\left(i_{2}\right),
$$

and

$$
\boldsymbol{x}^{\gamma(i)}=\left(x^{\gamma(i)}, y^{\gamma(i)}, z^{\gamma(i)}\right), \quad \gamma(i)=\gamma\left(i_{1}\right) \cdots \gamma\left(i_{2}\right) .
$$

If the junction area is enough small, the $z$ coordinate can be regarded as uniform in the $x y$ plane. We let the average of the $z$ coordinate of the mass points $\boldsymbol{x}^{\alpha(i)}$ and $\boldsymbol{x}^{\gamma(i)}$ be $\bar{z}^{\alpha \beta}$ and $\bar{z}^{\gamma \beta}$, which can be expressed as

$$
z^{\alpha \beta}=\frac{1}{\alpha\left(i_{2}\right)-\alpha\left(i_{1}\right)+1} \sum_{\alpha(i)=\alpha\left(i_{1}\right)}^{\alpha\left(i_{2}\right)} z^{\alpha(i)}
$$

and

$$
\bar{z}^{\gamma \beta}=\frac{1}{\gamma\left(i_{2}\right)-\gamma\left(i_{1}\right)+1} \sum_{\gamma(i)=\gamma\left(i_{1}\right)}^{\gamma\left(i_{2}\right)} z^{\gamma(i)} .
$$

From Eqs. (12) and (13), $U$ and $J$ of the rod resulting from stiffness $s_{\beta}$ and viscous damping $r_{\beta}$ are

$$
U_{\beta}=\frac{1}{2} s_{\beta}\left(\bar{z}^{\alpha \beta}-\bar{z}^{\gamma \beta}\right)^{2}
$$

and 


$$
J_{\beta}=\frac{1}{2} r_{\beta}\left(\dot{\bar{z}}^{\alpha \beta}-\dot{\bar{z}}^{\gamma \beta}\right)^{2}
$$

Substituting Eqs. (6), (7), (8), and (9) into Eqs. (4) and (5) gives

$$
\begin{gathered}
m_{\alpha(i) \alpha(j)} \ddot{q}^{\alpha(i)}+r_{\alpha(i) \alpha(j)} \dot{q}^{\alpha(i)}+s_{\alpha(i) \alpha(j)} q^{\alpha(i)} \\
+\frac{\partial}{\partial q^{\alpha(j)}} U^{\beta}+\frac{\partial}{\partial \dot{q}^{\alpha(j)}} J^{\beta}=f^{\alpha(i)}
\end{gathered}
$$

and

$$
\begin{gathered}
m_{\gamma(i) \gamma(j)} \ddot{q}^{\gamma(i)}+r_{\gamma(i) \gamma(j)} \dot{q}^{\gamma(i)}+s_{\gamma(i) \gamma(j)} q^{\gamma(i)} \\
+\frac{\partial}{\partial q^{\gamma(j)}} U^{\beta}+\frac{\partial}{\partial \dot{q}^{\gamma(j)}} J^{\beta}=0
\end{gathered}
$$

In Eqs. (16) and (17), the generalized coordinates for diaphragms 1 and $2, q^{\alpha(i)}$ and $q^{\gamma(i)}$, can be rewritten using the rectangular coordinate $\boldsymbol{x}^{\alpha(i)}$ and $\boldsymbol{x}^{\gamma(i)}$. The metric tensors of the mass, the stiffness, and the viscous damping can be regarded as constants. Only the equations in the $z$ direction are important since we take into account bending deflection. Thus $q^{\alpha(i)}$ and $q^{\gamma(i)}$ can be replaced with $z^{\alpha(i)}$ and $q^{\gamma(i)}$, respectively. Moreover, the metric tensors $m_{i j}, s_{i j}$, and $r_{i j}$ can be approximated as constants by Assumption 3 mentioned above.

Substituting Eqs. (14) and (15) into Eqs. (16) and (17) gives

$$
\begin{array}{r}
m_{\alpha z} \ddot{z}^{\alpha(i)}+r_{\alpha z} \dot{z}^{\alpha(i)}+s_{\alpha z} z^{\alpha(i)}+\delta_{\left(\alpha(i)=\alpha\left(i_{1}\right) \ldots \alpha\left(i_{2}\right)\right)} \\
\times\left[s_{\beta}\left(\bar{z}^{\alpha \beta}-\bar{z}^{\gamma \beta}\right)+r_{\beta}\left(\dot{\bar{z}}^{\alpha \beta}-\dot{\bar{z}}^{\gamma \beta}\right)\right]=f_{z}^{\alpha(i)}
\end{array}
$$

and

$$
\begin{gathered}
m_{\gamma z} \ddot{z}^{\gamma(i)}+r_{\gamma z} \dot{z}^{\gamma(i)}+s_{\gamma z} z^{\gamma(i)}-\delta_{\left(\gamma(i)=\gamma\left(i_{1}\right) \cdots \gamma\left(i_{2}\right)\right)} \\
\times\left[s_{\beta}\left(\bar{z}^{\alpha \beta}-\bar{z}^{\gamma \beta}\right)+r_{\beta}\left(\dot{\bar{z}}^{\alpha \beta}-\dot{\bar{z}}^{\gamma \beta}\right)\right]=0,
\end{gathered}
$$

where $\delta_{\left(\alpha(i)=\alpha\left(i_{1}\right) \cdots \alpha\left(i_{2}\right)\right)}$ and $\delta_{\left(\gamma(i)=\gamma\left(i_{1}\right) \cdots \gamma\left(i_{2}\right)\right)}$ are Kronecker deltas, which are equal to 1 if the mass points in diaphragms 1 and 2 belong to the junction area.

From infinitesimal deformation theory, the displacement in the $z$ direction is uniform. Therefore, Eqs. (18) and (19) can be rewritten in terms of deflection by defining the neutral plane as $z=0$. By letting the deflection be $\phi$, Eqs. (18) and (19) become

$$
\begin{aligned}
& m_{\alpha z} \ddot{\phi}^{\alpha(i)}+r_{\alpha z} \dot{\phi}^{\alpha(i)}+s_{\alpha z} \phi^{\alpha(i)}+\delta_{\left(\alpha(i)=\alpha\left(i_{1}\right) \cdots \alpha\left(i_{2}\right)\right)} \\
& \quad \times\left[s_{\beta}\left(\bar{\phi}^{\alpha \beta}-\bar{\phi}^{\gamma \beta}\right)+r_{\beta}\left(\dot{\bar{\phi}}^{\alpha \beta}-\dot{\bar{\phi}}^{\gamma \beta}\right)\right]=f_{z}^{\alpha(i)}
\end{aligned}
$$

and

$$
\begin{gathered}
m_{\gamma z} \ddot{\phi}^{\gamma(i)}+r_{\gamma z} \dot{\phi}^{\gamma(i)}+s_{\gamma z} \phi^{\gamma(i)}-\delta_{\left(\gamma(i)=\gamma\left(i_{1}\right) \cdots \gamma\left(i_{2}\right)\right)} \\
\times\left[s_{\beta}\left(\bar{\phi}^{\alpha \beta}-\bar{\phi}^{\gamma \beta}\right)+r_{\beta}\left(\dot{\bar{\phi}}^{\alpha \beta}-\dot{\bar{\phi}}^{\gamma \beta}\right)\right]=0 .
\end{gathered}
$$

The total deflection of diaphragms 1 and 2 is obtained by integrating Eqs. (20) and (21) throughout each diaphragm, yielding

$$
m_{\alpha z} \ddot{\Phi}^{\alpha}+r_{\alpha z} \dot{\Phi}^{\alpha}+s_{\alpha z} \Phi^{\alpha}
$$

$$
+\left[s_{\beta}\left(\bar{\Phi}^{\alpha \beta}-\bar{\Phi}^{\gamma \beta}\right)+r_{\beta}\left(\dot{\bar{\Phi}}^{\alpha \beta}-\dot{\bar{\Phi}}^{\gamma \beta}\right)\right]=F_{z}^{\alpha}
$$

and

$$
\begin{aligned}
& m_{\gamma z} \ddot{\Phi}^{\gamma}+r_{\gamma z} \dot{\Phi}^{\gamma}+s_{\gamma z} \Phi^{\gamma} \\
& \quad-\left[s_{\beta}\left(\bar{\Phi}^{\alpha \beta}-\bar{\Phi}^{\gamma \beta}\right)+r_{\beta}\left(\dot{\bar{\Phi}}^{\alpha \beta}-\dot{\bar{\Phi}}^{\gamma \beta}\right)\right]=0,
\end{aligned}
$$

where

$$
\begin{aligned}
& \Phi^{\alpha}=\sum_{\alpha(i)} \phi^{\alpha(i)}, \quad \Phi^{\gamma}=\sum_{\gamma(i)} \phi^{\gamma(i)}, \\
& \bar{\Phi}^{\alpha \beta}=\sum_{\alpha(i)=\alpha\left(i_{1}\right)}^{\alpha\left(i_{2}\right)} \phi^{\alpha(i)}, \text { and } \bar{\Phi}^{\gamma \beta}=\sum_{\gamma(i)=\gamma\left(i_{1}\right)}^{\gamma\left(i_{2}\right)} \phi^{\gamma(i)} .
\end{aligned}
$$

Equations (22) and (23) are ordinary differential equations (ODEs) including two functions, $\bar{\Phi}^{\alpha \beta}$ and $\bar{\Phi}^{\gamma \beta}$, that can be eliminated from the equations by assuming that the deflection in the junction area is multiplying the total deflection of diaphragms 1 and 2 by a constant. Namely, define the junction coefficients $\zeta_{\alpha}$ and $\zeta_{\gamma}$ as

$$
\bar{\Phi}^{\alpha \beta}=\zeta_{\alpha} \Phi^{\alpha} \text { and } \bar{\Phi}^{\gamma \beta}=\zeta_{\gamma} \Phi^{\gamma} .
$$

Accordingly, we substitute Eq. (25) into Eqs. (22) and (23) to get

$$
\begin{aligned}
& m_{\alpha z} \ddot{\Phi}^{\alpha}+r_{\alpha z} \dot{\Phi}^{\alpha}+s_{\alpha z} \Phi^{\alpha}+\left[s_{\beta}\left(\zeta_{\alpha} \Phi^{\alpha}-\zeta_{\gamma} \Phi^{\gamma}\right)\right. \\
& \left.+r_{\beta}\left(\zeta_{\alpha} \dot{\Phi}^{\alpha}-\zeta_{\gamma} \dot{\Phi}^{\gamma}\right)\right]=F_{z}^{\alpha}
\end{aligned}
$$

and

$$
\begin{aligned}
& m_{\gamma z} \ddot{\Phi}^{\gamma}+r_{\gamma z} \dot{\Phi}^{\gamma}+s_{\gamma z} \Phi^{\gamma}-\left[s_{\beta}\left(\zeta_{\alpha} \Phi^{\alpha}-\zeta_{\gamma} \Phi^{\gamma}\right)\right. \\
& \left.\quad+r_{\beta}\left(\zeta_{\alpha} \dot{\Phi}^{\alpha}-\zeta_{\gamma} \dot{\Phi}^{\gamma}\right)\right]=0 .
\end{aligned}
$$

Note that $\zeta_{\alpha}$ and $\zeta_{\gamma}$ are changed by the junction area and the position of the rod in the diaphragms.

In Eqs. (26) and (27), $m_{\alpha z}, m_{\gamma z}, s_{\alpha z}, s_{\gamma z}$, and $r_{\alpha z}$ are determined by the biharmonic equation of the diaphragms. In diaphragm 1, the biharmonic equations at the differential area element $d s$ in the junction area are represented by omitting $r_{\beta}$ as

$$
\begin{array}{r}
D_{\alpha} \nabla^{4} \phi^{\alpha} d s+\rho_{\alpha} h_{\alpha} \frac{d^{2}}{d t^{2}} \phi^{\alpha} d s \\
+\frac{E_{\beta}}{l_{\beta}}\left(\bar{\phi}^{\alpha \beta}-\bar{\phi}^{\gamma \beta}\right) d s=0,
\end{array}
$$

where $E_{\beta}$ is Young's modulus of the $\operatorname{rod}$, and $l_{\beta}$ is the height of the rod.

From Eq. (26), each lumped constant in the ODE is associated with $m_{\alpha z}=\rho_{\alpha} h_{\alpha}, s_{\alpha z}=D_{\alpha} \nabla^{4}$, and $s_{\beta}=E_{\beta} / l_{\beta}$ for diaphragm 1 and the rod. Similarly, these relational expressions hold for diaphragm 2. Equations (26) and (27) can be expressed by the lumped constant equivalent circuit shown in Fig. 5.

In Fig. 5, there are two resonant loops, i.e., loops 1 and 2. Loop 1 is a series resonance loop including diaphragms 1 and 2. This resonance causes the first resonant peak of the current. Loop 2 comprises two parallel resonance circuits 


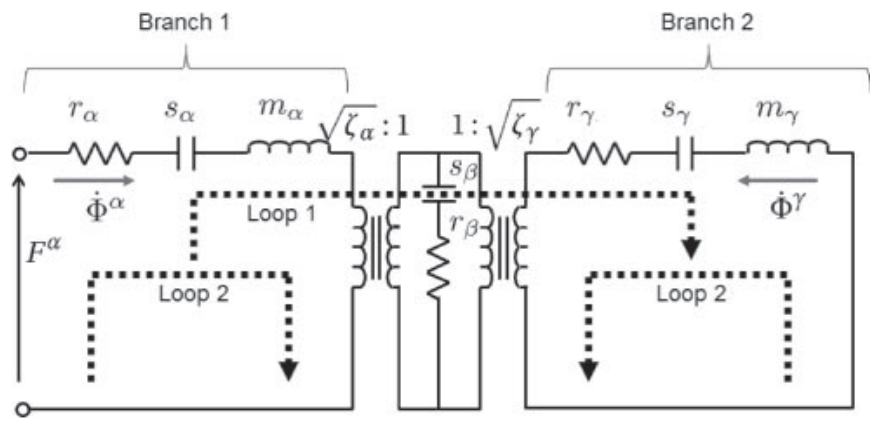

Fig. 5 Equivalent circuit of mechanical components of transducer having double-linked diaphragms.

including the rod, diaphragm 1 , and diaphragm 2. This resonance causes the second resonant peak of the current.

Branches 1 and 2 represent diaphragms 1 and 2. Thus, the vibration displacement of each diaphragm is dominated by the voltage applied to each branch, which is equal to the applied pressure. In Fig. 5, the phase of the current represents the phase of the vibration velocity of the diaphragm. At the first resonant frequency (loop 1), the currents in branches 1 and 2 are the same. By contrast, at the second resonant frequency (loop 2), they are opposite to each other. The vibration direction will be confirmed by experimental results from a laser Doppler vibrometer (LDV) later.

Note that the resonant frequencies of loop 1 , branch 1 and branch 2 are

$$
\begin{gathered}
f_{11}=\frac{1}{2 \pi} \sqrt{\frac{s_{\alpha z}+s_{\gamma z}}{m_{\alpha z}+m_{\gamma z}}}, \quad f_{\mathrm{b} 1}=\frac{1}{2 \pi} \sqrt{\frac{s_{\alpha z}}{m_{\alpha z}}} \\
\text { and } f_{\mathrm{b} 2}=\frac{1}{2 \pi} \sqrt{\frac{s_{\gamma z}}{m_{\gamma z}}} .
\end{gathered}
$$

The negative peak of the applied force to each diaphragm around the first resonant peak caused by the resonance of branches 1 and 2, respectively. The magnitude correlation of the resonant frequencies $f_{11}, f_{\mathrm{b} 1}$ and $f_{\mathrm{b} 2}$, i.e. the sequence of the negative peaks and the first resonant peak is determined by the stiffness and the mass of diaphragms 1 and 2 .

The negative peak of the applied force of diaphragm 2 (branch 2) tends to the sound pressure level because diaphragm 2 is closer to the observation point than diaphragm 1. However, the negative peak does not necessarily appear in the sound pressure level.

\subsection{Solution of the Biharmonic Equation of the Bending Disk}

In this section, we discuss the biharmonic equation of the bending plate for diaphragms 1 and 2 . The detailed calculation is described in the Appendix [12].

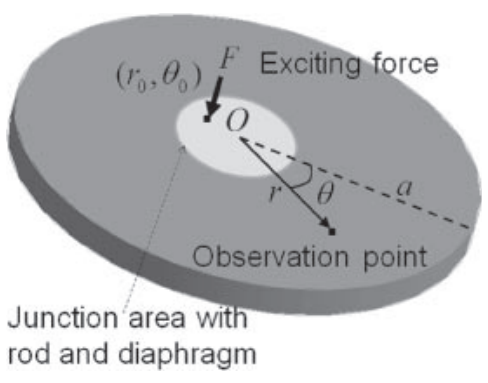

Fig. 6 Schematic of disk-shaped diaphragm and exciting force.

Figure 6 shows the model of the disk-shaped diaphragm. The radius of the diaphragm is $a$, its mass density is $\rho$, and the viscous damping is $c$. We let its coordinates in the cylindrical coordinate system be $(r, \theta, z)$ and define the coordinates of the concentrated exciting force as $\left(r_{0}, \theta_{0}, h\right)$.

The biharmonic equation of undamped vibration for harmonic excitation is expressed as

$$
D \nabla^{4} \phi-\rho h \frac{d^{2} \phi}{d t^{2}}=\frac{f}{r} \delta\left(\boldsymbol{r}-\boldsymbol{r}_{0}\right) e^{j \omega t},
$$

where $f$ is the magnitude of the exciting force, $\omega$ is the angular frequency of the exciting force, and $D$ is the bending stiffness.

The bending stiffnesses of diaphragms 1 and 2 are calculated in the Appendix. The bending stiffness of diaphragm 2 is calculated from Eq. (A-2) by using the stiffness and the mass density of the metal and the piezoelectric element. The excitation force exerted by the piezoelectric element is calculated by the boundary condition for diaphragm 1, which is written as Eqs. (A.9) and (A.10).

The general solution of the homogeneous equation (30) for vibration around the fundamental natural frequency is represented by using the wave number for the fundamental natural mode $\kappa_{0}$ as

$$
\phi_{\mathrm{g}} \simeq A_{0} J_{0}\left(\kappa_{0} r\right)+B_{0} I_{0}\left(\kappa_{0} r\right)
$$

The particular solution of Eq. (30) is obtained as the sum of the weak solution for each excitation force using the wave number $\kappa$ :

$$
\begin{aligned}
\phi_{\mathrm{p}}= & -\frac{1}{8 \kappa^{2}} \iint\left[Y_{0}\left(\kappa\left|\boldsymbol{r}-\boldsymbol{r}_{0}\right|\right)\right. \\
& \left.+\frac{2}{\pi} K_{0}\left(\kappa\left|\boldsymbol{r}-\boldsymbol{r}_{0}\right|\right)\right] r_{0} d r_{0} d \theta_{0} .
\end{aligned}
$$

The general solution of Eq. (30) is expressed as the sum of Eqs. (31) and (32) as $\phi=\phi_{\mathrm{g}}+\phi_{\mathrm{p}}$.

As the length of the side of the rod increases, the excitation points $\boldsymbol{r}_{0}$ increase. A particular solution $\phi_{\mathrm{p}}$ is added for each excitation point. Therefore, the vibration forms of the diaphragms vary according to the increase of 
Table 1 Dimensions of diaphragm and rod.

\begin{tabular}{|c|c|c|c|}
\hline \multicolumn{2}{|c|}{ Diaphragm } & PZT & Rod \\
\hline Radius & Thickness & Thickness & Height $=$ Side \\
\hline $7.5 \mathrm{~mm}$ & $1.55 \mathrm{~mm}$ & $0.1 \mathrm{~mm}$ & $\begin{array}{l}2.0-5.0 \mathrm{~mm} \text { (experiment) } \\
1.0-5.0 \mathrm{~mm} \text { (calculation) }\end{array}$ \\
\hline
\end{tabular}

Table 2 Physical properties of the diaphragm.

\begin{tabular}{ccc}
\hline Material & Mass density & Young's modulus \\
\hline phosphor bronze & $8.80 \times 10^{3} \mathrm{~kg} / \mathrm{m}^{3}$ & $1.10 \times 10^{2} \mathrm{GPa}$ \\
\hline
\end{tabular}

the rod size from the general solution $\phi_{\mathrm{g}}$. This variation of the vibration form results in variation of the junction coefficients $\zeta_{\alpha}$ and $\zeta_{\gamma}$.

The ratio of the coefficients $A_{0}$ and $C_{0}$ in Eq. (31) are determined by the boundary condition of the bending plate and by using the Rayligh-Ritz method. In diaphragm 1, by Eq. (31), the junction coefficient $\zeta_{\alpha}$ is calculated as

$$
\zeta_{\alpha}=\frac{\bar{\Phi}^{\alpha \beta}}{\Phi^{\alpha}}, \quad \Phi^{\alpha}=\int_{0}^{2 \pi} \int_{0}^{a_{\alpha}} \phi^{\alpha} r d r d \theta,
$$

and

$$
\bar{\Phi}^{\alpha \beta}=\int_{0}^{2 \pi} \int_{0}^{\eta_{\alpha}} \phi^{\alpha} r d r d \theta,
$$

where $a_{\alpha}$ is the radius of the diaphragm 1 and $\eta_{\alpha}$ is the radius of the cross section of the rod.

From Eqs. (31), (32), and (33), we see that $\Phi^{\alpha}, \bar{\Phi}^{\alpha \beta}$, $\zeta_{\alpha}$, and $\zeta_{\gamma}$ are functions of the angular frequency of the exciting force, $\omega$, and the dimensions of the rod (see the Appendix). When the rod has a rectangular shape, $\zeta_{\alpha}$ and $\zeta_{\gamma}$ are calculated by converting Eq. (33) into a rectangular coordinate system.

\subsection{Numerical Calculation}

We derive the design condition to improve the frequency response of transducers by numerical calculations.

First, we show the production conditions of the prototypes, which are used to numerical calculation. The dimensions and the mechanical parameters of the transducer component are listed in Tables 1, 2, and 3.

The elastic supports were fixed at a position of $5.0 \mathrm{~mm}$, which is the node of the fundamental natural vibration. The piezoelectric elements were fabricated by Nihon Ceratec Co., Ltd. (Material code C). Detailed information, such as the elasto-piezo-dielectric (EPD) matrix, is listed in Table 4.

When the minimum length of the side of the rods is $<3.00 \mathrm{~mm}$, maintaining the directivity of the cuboid or cylindrical rod while bonding the diaphragms and the rod
Table 3 Physical properties of the rod and elastic support.

\begin{tabular}{cccc}
\hline Part & Material & $\begin{array}{c}\text { Mass } \\
\text { density }\end{array}$ & $\begin{array}{c}\text { Young's } \\
\text { modulus }\end{array}$ \\
\hline rod & acrylic resin & $1.19 \times 10^{3} \mathrm{~kg} / \mathrm{m}^{3}$ & $3.14 \mathrm{GPa}$ \\
\hline elastic support & polychloroprene & $1.20 \times 10^{3} \mathrm{~kg} / \mathrm{m}^{3}$ & $10.00 \mathrm{MPa}$ \\
\hline
\end{tabular}

Table 4 Physical properties of the piezoelectric board.

\begin{tabular}{cccc}
\hline $\begin{array}{c}\text { Dielectric } \\
\text { constant }\end{array}$ & $\begin{array}{c}\text { Coupling } \\
\text { factor }\end{array}$ & $\begin{array}{c}\text { Piezoelectric } \\
\text { strain constant } \\
\times 10^{-12} \mathrm{~m} / \mathrm{V}\end{array}$ & $\begin{array}{c}\text { Elastic } \\
\text { constant } \\
\times 10^{-12} \mathrm{~m}^{2} / \mathrm{N}\end{array}$ \\
\hline$\epsilon_{33} / \epsilon_{0}=4,500$ & $K_{\mathrm{r}}=0.61$ & $d_{31}=-160$ & $S_{11}=15.2$ \\
$\epsilon_{11} / \epsilon_{0}=4,700$ & $K_{31}=0.35$ & $d_{33}=280$ & $S_{33}=15.5$ \\
& $K_{33}=0.65$ & $d_{15}=450$ & \\
\hline
\end{tabular}

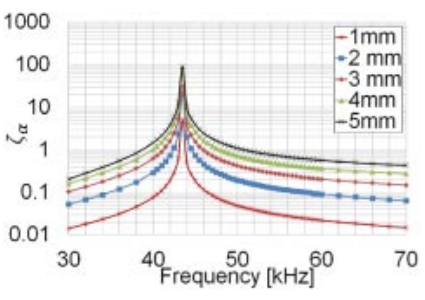

(a) Junction coefficient of diaphragm 1

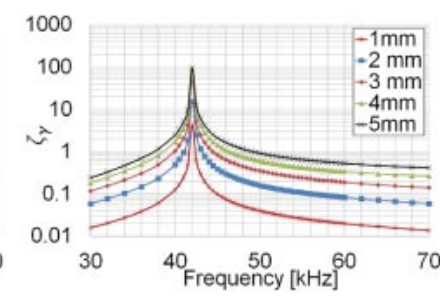

(b) Junction coefficient of diaphragm 2
Fig. 7 Calculation results for the junction coefficient for four types of rods with side lengths of (a) diaphragm 1 and (b) diaphragm 2.

becomes difficult. For this reason, the rod and the elastic supports were cubically shaped.

We chose acryl as the material for the cubic rod because square bars of acryl of various sizes were easy to obtain on the market. It was difficult to make acryl cubes whose side length is $1 \mathrm{~mm}$. However, the calculation results for 1-mm cubic rod are shown to clarify the trend of the vibration of the sound pressure level.

The elastic supports made of chloroprene rubber are located at the nodes of the fundamental resonance mode of diaphragm 1.

Figure 7 shows the calculation results of $\zeta_{\alpha}$ and $\zeta_{\gamma}$ for each length of one side of the rod. As shown in Fig. 7, the junction coefficients increase according to the increase of the length of the side of the rods. This increase of the junction coefficient induces the increase of the frequency gap between the two resonant peaks.

The vibration form is calculated by using the following process:

1. Calculate the exciting force at the rod by using Eqs. (26) and (27).

2. Calculate the coefficients of the general solution (31) by using the Rayligh-Ritz method (see the Appendix). 
Air (Boundary: Mur first order absorbing boundary)

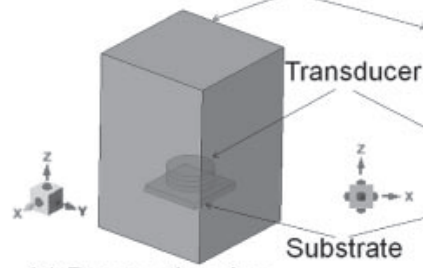

(a) Perspective view

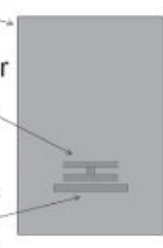

(b) Side View

Fig. 8 Schematic of FDTD model: (a) perspective view; (b) side view.

Table 5 Dimensions used for the FDTD calculation.

\begin{tabular}{cccc}
\hline Time step & Grid size & Size of space & $\begin{array}{c}\text { Size of } \\
\text { substrate }\end{array}$ \\
\hline & & $1.0 \mathrm{~mm}(x y$ plane $)$ & $x: 4 \mathrm{~cm}$ \\
$0.6 \mu \mathrm{s}$ & $1.0 \mathrm{~mm}(x y$ plane $)$ & $0.5 \mathrm{~mm}(z$ axis $)$ & $z: 6 \mathrm{~cm}$ \\
\hline
\end{tabular}

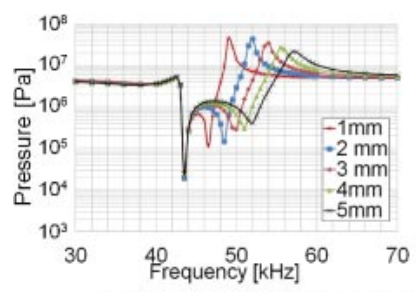

(a) Pressure applied to diaphragm 1

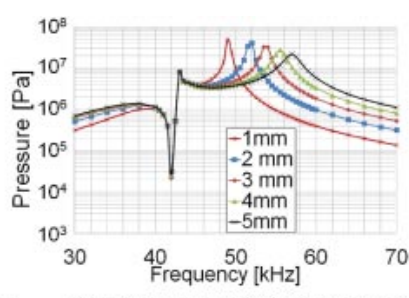

(b) Pressure applied to diaphragm 2

Fig. 9 Pressure applied to (a) diaphragm 1 and (b) diaphragm 2.

3. Calculate the sound pressure level at $3 \mathrm{~cm}$ from the center of the upper surface of diaphragm 2 by using the the finite difference time domain (FDTD) method.

4. Calculate the sound pressure level at $30 \mathrm{~cm}$ from the center of the upper surface of diaphragm 2 by assuming spherical diffusion because the Rayleigh length of the sound wave $(30-70 \mathrm{kHz})$.

Figure 8 shows the FDTD calculation model. Table 5 lists details of the FDTD model.

Figures 9(a) and 9(b) show the pressures applied to diaphragms 1 and 2, which are equivalent to the voltages applied to branches 1 and 2 in the equivalent circuit (Fig. 5), when $Q=500$ and $r_{\beta}=25 r_{\alpha}$. The sound pressure level of the sound radiated by the transducers is almost dominated by the applied pressures as shown in Figs. 9(a) and 9(b). As shown in Figs. 9(a) and 9(b), the magnitude of the second resonant peak decreases according to the increase of the length of the side of the rod. Moreover, the second resonant frequency increases according to the increase of the sizes of the rod. These trends are common to the frequency response of the sound pressure level, as shown in Figs. 10 and 11.

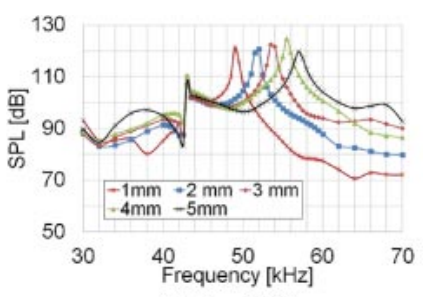

(a) $Q=1000$

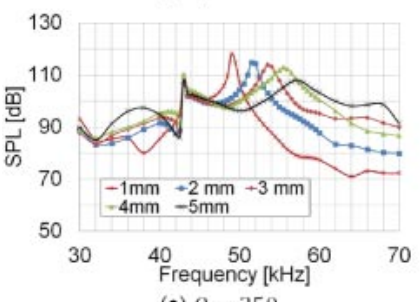

(c) $Q=250$

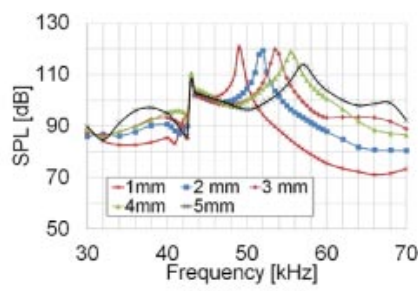

(b) $Q=500$

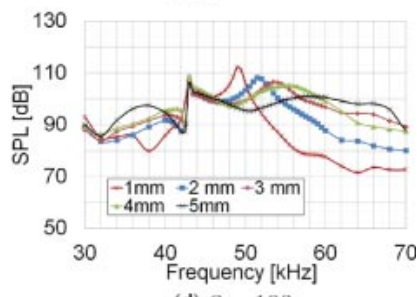

(d) $Q=100$
Fig. 10 Frequency response of the sound pressure level for (a) $Q=1,000$, (b) $Q=500$, (c) $Q=250$, and (d) $Q=100$.
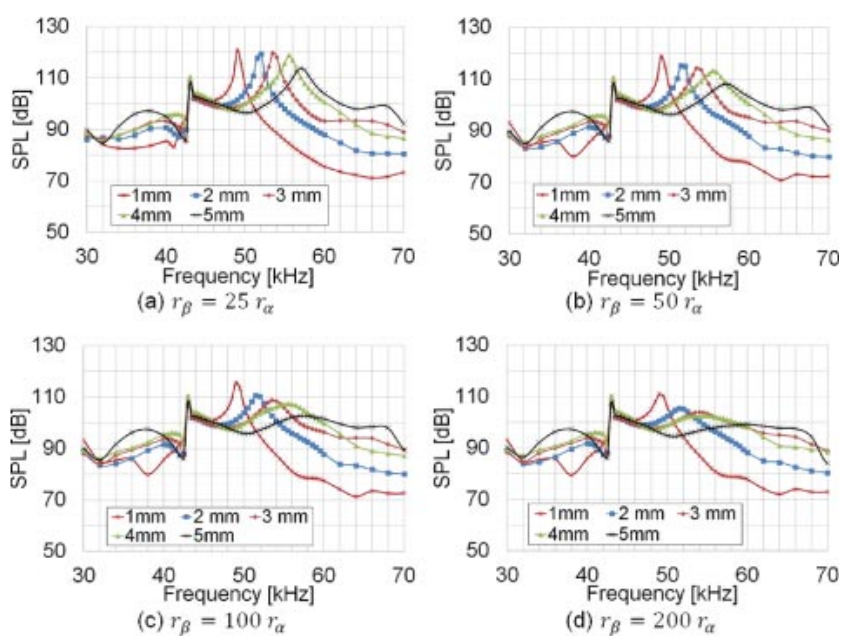

Fig. 11 Frequency response of the sound pressure level for (a) $r_{\beta}=25 r_{\alpha}$, (b) $r_{\beta}=50 r_{\alpha}$, (c) $r_{\beta}=100 r_{\alpha}$, and (d) $r_{\beta}=200 r_{\alpha}$.

Figure 10 shows the calculation results for the frequency response of the sound pressure level. In Fig. 10 the quality factors for the diaphragm are $Q=1,000,500,250$, and 100. The sound pressure level of the two resonant peaks decreases according to the decrease of the quality factor.

Figure 11 shows the influence of viscous damping of the rod, $r_{\beta}$, on the sound pressure level. The sound pressure level of the second resonance decreases according to the increases of $r_{\beta}$. However, the first resonant peak is constant, independently of $r_{\beta}$. From these results we conclude that only the second resonance mode, i.e., loop 2 of the equivalent circuit, includes the viscous damping of the rod, $r_{\beta}$.

To make use of the second resonant peak, the stiffness of the rod, $r_{\beta}$, the junction coefficients $\zeta_{\alpha}$ and $\zeta_{\gamma}$ must be suppressed more than 1-mm cubic rod. Figure 12 shows the 


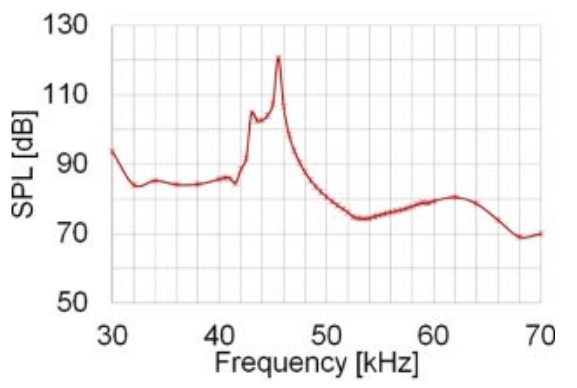

Fig. 12 Target frequency response of the sound pressure level $\left(r_{\beta}=25 r_{\alpha}, Q=500\right)$.

frequency response of the sound pressure level of the target prototype, whose rod size is $0.5 \mathrm{~mm}$ (depth) $\times 0.5 \mathrm{~mm}$ (width) $\times 1.5 \mathrm{~mm}$ (height). Additionally, Q is set to 500 , $r_{\beta}$ is set to $25 r_{\alpha}$. As shown in Fig. 12, the sound pressure level of the target prototype has the large second resonant peak, which is widened by the first resonant peak.

From the calculation results shown in Figs. 9, 10, and 11, the design condition to form high and wide resonant peak of the sound pressure level, i.e., the target characteristic as shown in Fig. 12, can be summarized as follows: 1. Suppress the stiffness of the rod, $r_{\beta}$, and the junction coefficients $\zeta_{\alpha}$ and $\zeta_{\gamma}$ to shrink the frequency gap between the two resonant peaks and increase the magnitude of the second resonant peak.

2. Suppress the viscous damping of the rod, $r_{\beta}$, to increase the magnitude of the second resonant peak.

\section{EXPERIMENTS}

\subsection{Experimental Results}

The experimental results are shown to confirm the derived design condition by using the calculation results. The production conditions of the prototypes are listed in Tables 1, 2, 3, and 4 .

Figure 16 shows the prototype transducers for the experiments.

First, we show the vibration velocity obtained by LDV to explain the vibration form and the direction of diaphragms 1 and 2. The measured transducer has a $5 \mathrm{~mm}$ cubic rod. The input voltage to the transducers was $1 \mathrm{~V}_{\text {rms. }}$. The vibration velocities of diaphragms 1 and 2 were measured at the bottom surface and top surface, respectively, as shown in Figs. 13(a) and 13(b). The measured surface of diaphragms 1 and 2 are opposite to each other. Therefore, when the vibration directions of the two diaphragms are the same, their vibration velocities are out of phase by $180 \mathrm{deg}$. Figures 14 and 15 show the vibration velocity at the first $(43.4 \mathrm{kHz})$ and second $(57.5 \mathrm{kHz})$ resonant frequencies, respectively. As shown in Figs. 14(a) and 14(b), the vibration directions of the two diaphragms are the same at the first resonant frequency; i.e., the phase difference is $\sim 0 \mathrm{deg}$. In contrast, at the (a) Diaphragm 1

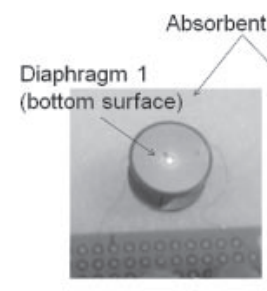

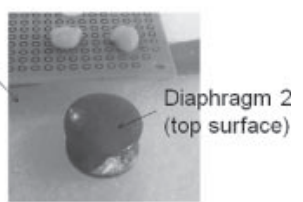

(b) Diaphragm 2
Fig. 13 Photographs of measured surface by LDV for (a) diaphragm 1 (bottom surface) and (b) diaphragm 2 (top surface).

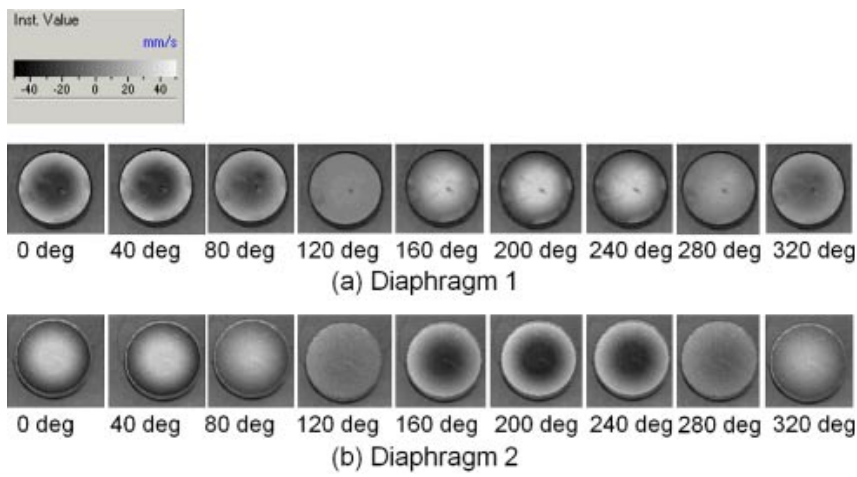

Fig. 14 Photographs of measured results of vibration velocity at the first resonant frequency $(43.4 \mathrm{kHz})$ by LDV for (a) diaphragm 1 (bottom surface) and (b) diaphragm 2 (top surface).

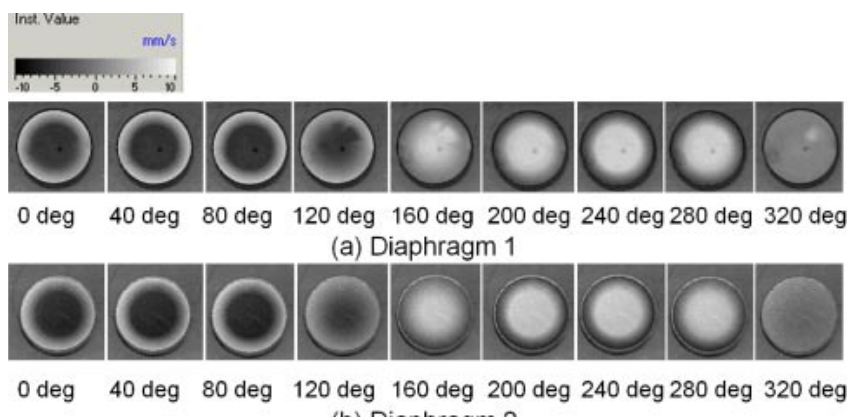

(b) Diaphragm 2

Fig. 15 Photographs of measured results of vibration velocity at the second resonant frequency $(57.5 \mathrm{kHz})$ by LDV for (a) diaphragm 1 (bottom surface) and (b) diaphragm 2 (top surface).

second resonant frequency, the vibration directions are opposite to each other; i.e., the phase difference is $\sim 180 \mathrm{deg}$. The vibration forms of the two diaphragms are that of the first natural mode, which can be described as the general solution of the biharmonic equation Eq. (31). As mentioned above, the vibration form is almost determined by the general solution $\phi_{\mathrm{g}}$.

Figure 17 shows system used for measuring the sound pressure level. 


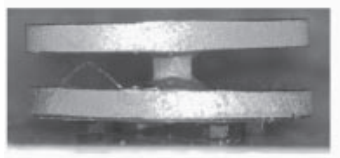

(a) $b=2.0[\mathrm{~mm}]$

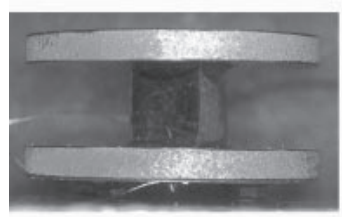

(c) $b=4.0[\mathrm{~mm}]$

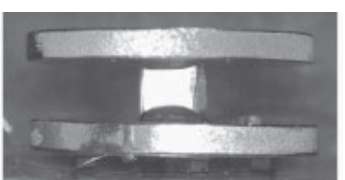

(b) $b=3.0[\mathrm{~mm}]$

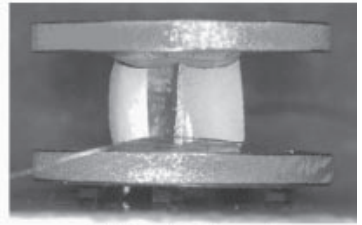

(d) $\mathrm{b}=5.0[\mathrm{~mm}]$
Fig. 16 Photographs of the transducer with four types of rods with side lengths of (a) 2.0, (b) 3.0, (c) 4.0, and (d) $5.0 \mathrm{~mm}$.

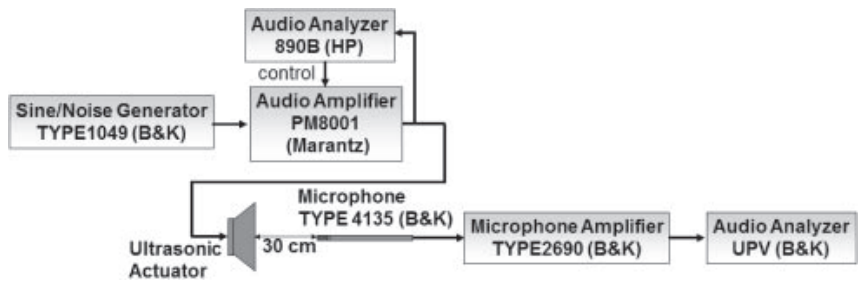

Fig. 17 Schematic of the system used for measuring the sound pressure level.

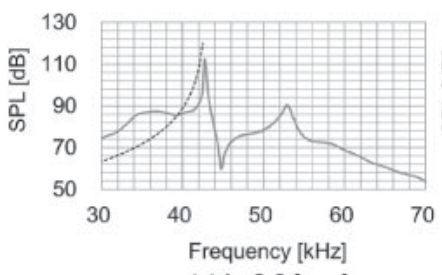

(a) $b=2.0[\mathrm{~mm}]$

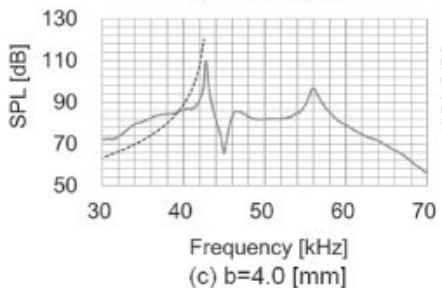

(c) $b=4.0[\mathrm{~mm}]$

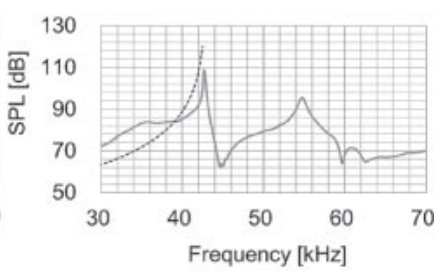

(b) $b=3.0[\mathrm{~mm}]$

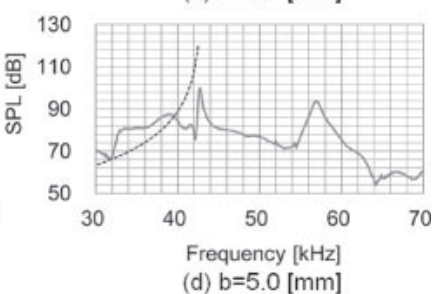

Fig. 18 Measured sound pressure level of the transducer with four types of rods with side lengths of (a) 2.0, (b) 3.0 , (c) 4.0 , and (d) $5.0 \mathrm{~mm}$.

Figure 18 shows the frequency response of the sound pressure level. The input voltage was $5 \mathrm{~V}_{\mathrm{rms}}$ and the measuring distance was $30 \mathrm{~cm}$. We confirmed that the first resonant peak does not vary and that the second resonant peak shifts to the higher frequency side according to the increase in the length of one side, which are equivalent to the increase in $\zeta_{\alpha}$ and $\zeta_{\gamma}$. Figure 7 shows the variation in the frequency gap between the two resonant peaks.
Table 6 Electrical characteristics of transducers at resonant frequencies with a cubic rod.

\begin{tabular}{ccccc}
\hline \multirow{2}{*}{ Side of rod } & $\begin{array}{c}\text { Resonant } \\
\text { number }\end{array}$ & $\begin{array}{c}\text { Resonant } \\
\text { frequency }\end{array}$ & $Q$ factor & Conductance \\
\hline $2.0 \mathrm{~mm}$ & first & $42.9 \mathrm{kHz}$ & 571.7 & $3.79 \times 10^{-2} \mathrm{~S}$ \\
\cline { 2 - 5 } & second & $53.2 \mathrm{kHz}$ & 68.2 & $4.20 \times 10^{-3} \mathrm{~S}$ \\
\hline \multirow{2}{*}{$3.0 \mathrm{~mm}$} & first & $42.8 \mathrm{kHz}$ & 356.9 & $2.74 \times 10^{-2} \mathrm{~S}$ \\
\cline { 2 - 5 } & second & $55.1 \mathrm{kHz}$ & 70.6 & $4.72 \times 10^{-3} \mathrm{~S}$ \\
\hline \multirow{2}{*}{$4.0 \mathrm{~mm}$} & first & $42.9 \mathrm{kHz}$ & 357.6 & $2.61 \times 10^{-2} \mathrm{~S}$ \\
\cline { 2 - 5 } & second & $56.2 \mathrm{kHz}$ & 68.2 & $4.63 \times 10^{-3} \mathrm{~S}$ \\
\hline $5.0 \mathrm{~mm}$ & first & $42.8 \mathrm{kHz}$ & 237.9 & $1.37 \times 10^{-2} \mathrm{~S}$ \\
\cline { 2 - 5 } & second & $57.2 \mathrm{kHz}$ & 64.7 & $3.53 \times 10^{-3} \mathrm{~S}$ \\
\hline
\end{tabular}

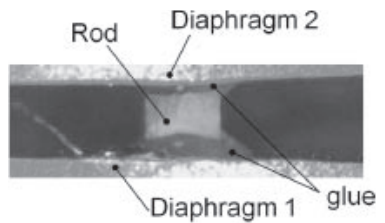

Fig. 19 Photograph of the bonding part of the transducer with a standard rod with a side length of $2.0 \mathrm{~mm}$.

The magnitudes of the second resonant peaks are small in Fig. 18. The degradation of the second resonant peaks is caused by the wide frequency gap between the two resonant frequencies and the large viscous damping of the $\operatorname{rod}, r_{\beta}$, which are described by the calculation results. It is confirmed that viscous damping of the rod, $r_{\beta}$, is large by inspection of the electrical characteristics listed in Table 6. The $Q$ factors of the second resonant frequencies are small for all prototypes. The increase in viscous damping of the rod, $r_{\beta}$, is caused by the increase of the rod itself and the increase of the glue. Figure 19 shows a photograph of the bonding part $(b=2.0 \mathrm{~mm})$. A large quantity of glue is applied around the rod. This is why it is difficult to ensure flatness of the bonding surface for either a cubic or cylindrical rod. The upper and lower surfaces of the rod are not flexible because the stiffness of the rod is high.

In Fig. 18, the dotted lines show the criterion used to evaluate whether the sound pressure level of the transducers satisfies the ideal frequency response, i.e., the inverse response of the sound pressure level of demodulated sound expressed by Eq. (1). This criterion to evaluate transducer performance was determined by considering the following conditions:

1. The input signal used was the lower sideband modulation (LSB) and the carrier wave.

2. The frequency of carrier wave, $f_{\mathrm{c}}$, was set to one of the resonant frequencies (higher resonant peak). 


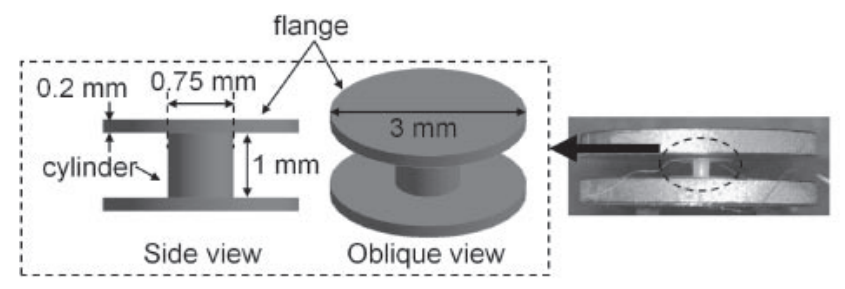

(a) Picture of bobbin structured rod

(b) Picture of transducer

Table 7 Electrical characteristics at resonant frequencies of transducers with a bobbin-structured rod.

\begin{tabular}{cccc}
\hline $\begin{array}{c}\text { Resonant } \\
\text { number }\end{array}$ & $\begin{array}{c}\text { Resonant } \\
\text { frequency }\end{array}$ & $Q$ factor & Conductance \\
\hline first & $42.8 \mathrm{kHz}$ & 950.4 & $2.64 \times 10^{-2} \mathrm{~S}$ \\
\hline second & $45.0 \mathrm{kHz}$ & 333.8 & $3.70 \times 10^{-2} \mathrm{~S}$ \\
\hline
\end{tabular}

Fig. 20 (a) Schematic and (b) photograph of the transducer with a bobbin-structured rod.

3. The bandwidth of the demodulated sound should be between $500 \mathrm{~Hz}$ and $10 \mathrm{kHz}$.

4. The target sound pressure level should be $120 \mathrm{~dB}$ SPL at $f_{\mathrm{c}}-500 \mathrm{~Hz}$.

From condition 1, the sound pressure level in the frequency range lower than the carrier frequency $f_{\mathrm{c}}$ is focused. Condition 3 is due to the difficulty in demodulating low-frequency sound. Condition 4 is invoked so that the sound pressure level of each transducer reaches the threshold level of the second harmonic wave generation at a distance of $<2 \mathrm{~m}$ [13].

If a measured sound pressure level is much lower than the level indicated by the dotted lines, the transducer's input voltage needs to be significantly boosted to satisfy the ideal frequency response. In the frequency range between the resonant frequencies $(42.7-39.0 \mathrm{kHz})$, the measured frequency responses (a)-(d) are much lower than those indicated by the dotted lines.

\subsection{Improvement of Structure}

The solutions to achieve the target frequency response of the sound pressure level, mentioned in the previous section are as follows:

1. Suppress the excess increase in the equivalent stiffness and viscous damping by adjusting $\zeta_{\alpha}$ and $\zeta_{\gamma}$.

2. By expanding the area of the bonding surface, stabilize the bonding with a small amount of glue.

For solutions to 1 and 2, we propose a bobbinstructured rod, as shown in Fig. 20.

It is difficult to exactly control the radius because the cylinder part must be processed by micromachining. Therefore, the radius of the cylinder part is the finished dimension.

The bobbin-structured rod is made of acrylonitrile butadiene styrene (ABS) copolymer resin because of the ease with which it can be processed. It was difficult to fabricate the micro bobbin-structured rod by using acrylic resin, because this material easily cracked in the machining process. Therefore we chose ABS resin as the material of the rod.

The bonding surface is expanded and given flexibility by using two thin flange parts. The excess increase in $\zeta_{\alpha}$

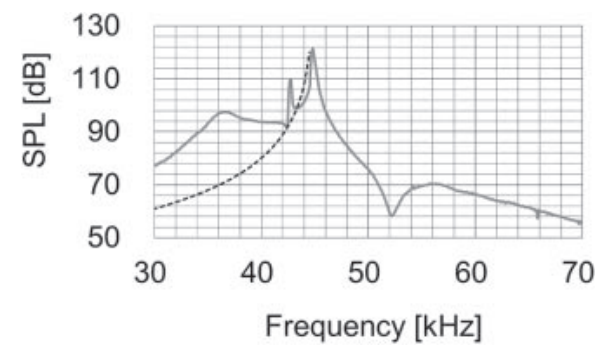

Fig. 21 Measured sound pressure level of the improved transducer with a bobbin-structured rod.

and $\zeta_{\gamma}$ can be suppressed because the bonding stability is ensured with a small amount of glue and the cylinder part of the rod can be narrow. This rod structure has many advantages, such as the ease of manufacturability of the rod itself and its stability during the adhesive curing process. Table 7 lists the electrical characteristics and Fig. 21 shows the frequency response of the sound pressure level of the bobbin-structured rod.

Figure 21 is close to the calculated target sound pressure level Fig. 12. This transducer has a large second peak; therefore, the carrier frequency can be set to the second resonant frequency. Accordingly, we drew the dotted line for the ideal frequency response from the second resonant frequency. The conductance and $Q$ factor of the second resonant peak greatly improved and the frequency gap between the first and second resonant peaks decreased to $2.2 \mathrm{kHz}$.

In the frequency range of the second resonant frequency $(45.0-42.0 \mathrm{kHz})$, the measured frequency response is close to that indicated by the dotted line. In the frequency range lower than $42.0 \mathrm{kHz}$, the measured frequency response is higher than that indicated by the dotted line. This result shows that the improved transducer almost satisfies the ideal frequency response without high-gain boost.

\section{CONCLUSIONS}

Through a theoretical analysis and the use of experiments, we presented a systematic design of an ultrasonic piezoelectric transducer for parametric speakers with double-linked diaphragms. We focused on controlling the resonant frequency and the magnitude of the resonant 
peaks while maintaining the mechanical quality factor as an approach to achieve the ideal frequency response of the sound pressure. The ideal frequency response of the sound pressure level was defined as the inverse frequency response of the sound pressure of the self-demodulation sound of the AM wave, i.e., $-12 \mathrm{~dB} /$ octave, passing through the neighborhood of the resonant peak used as the carrier frequency.

It is important to utilize the second resonant peak to form the ideal frequency response. We established a design method to make use of the second resonant peak. Namely, we clarified that the most important design factors are restraints on the rod size and the high stability of the bonding of the rod. The theoretical calculation results indicated that narrowed rods contribute to the ideal frequency response of the sound pressure level having the widened and enhanced peak.

By using the design information, with due consideration of manufacturability, an improved transducer structure was proposed. The key part of the improved transducer is the bobbin-structured rod, which has two thin flanges. The bobbin-structured rod ensures the bonding stability and restraints on the diameter of the rod. The improved transducer nearly satisfies the ideal frequency response of the sound pressure level.

\section{REFERENCES}

[1] P. J. Westervelt, "Parametric acoustic array," J. Acoust. Soc. Am., 35, 535-537 (1963).

[2] M. Yoneyama and J. Fujimoto, "The audio spotlight: An application of nonlinear interaction of sound waves to a new type of loudspeaker design," J. Acoust. Soc. Am., 73, 15321536 (1983).

[3] T. Kamakura, M. Yoneyama and K. Iketani, "Study for practical application of parametric speaker," J. Acoust. Soc. Jpn. (J), 41, 378-385 (1985) (in Japanese).

[4] S. Takeoka and Y. Yamasaki, "Acoustic projector using directivity controllable parametric loudspeaker array," Proc. ICA 2010, p. 921 (2010).

[5] T. Ikeda, Fundamentals of Piezoelectricity (Oxford University Press, New York, 1990), 280 pp.

[6] S. Takahashi and S. Hirose, "Vibration-level characteristics of lead zirconate titanate ceramics," Jpn. J. Appl. Phys., 31, 3055-3057 (1992).

[7] S. Sakai, Y. Shiozawa and T. Toi, "Clarification of sound radiation mechanism for airborne ultrasonic transducer," Acoust. Sci. \& Tech., 30, 404-409 (2009).

[8] J. Kuroda, Y. Onishi, M. Komoda, Y. Oikawa and Y. Yamasaki, "Design of resonant frequencies of the piezoelectric actuator with integrated components," Proc. ICA (POMA Vol. 19), pp. 030-066 (2013).

[9] R. Holland, "Analysis of multiterminal piezoelectric plates," J. Acoust. Soc. Am., 41(4B), 940-952 (1967).

[10] J. Soderkvist, "An equivalent circuit description of two coupled vibrations," J. Acoust. Soc. Am., 90, 663-699 (1991).

[11] J. Saneyoshi, Y. Kikuchi and O. Nomoto, Handbook of Ultrasonic Technologies (Nikkan Kogyo Shimbun, Tokyo, 1978), Chap. 3, pp. 381-388 (in Japanese).

[12] Y. Urata, "Analysis of the bending plate by the analytical solutions," Trans. Jpn. Soc. Mech. Eng. Ser. C, 68(668), 10361041 (2002) (in Japanese).

[13] J. A. Gallego-Juarez and L. Gaete-Garreton, "Propagation of finite-amplitude ultrasonic waves in air-I. Spherically driving waves in the free field," J. Acoust. Soc. Am., 73, 761-766 (1983).

[14] T. Suzuki, M. H. Isaka and K. Suzuki, "Vibration characteristics of unimorph type circular piezoelectric vibrator," Dynamic and Design Conference 2007, No. 07-08 (2007) (in Japanese).

\section{APPENDIX: CALCULATION OF THE BIHARMONIC EQUATION OF THE BENDING DISK}

In Eq. (30), the bending stiffness of diaphragm $1, D$, is expressed as

$$
D=\frac{E h^{3}}{12\left(1-v^{2}\right)} .
$$

In contrast, diaphragm 2 consists of the metal and the piezoelectric element, so its bending stiffness is [14]

$$
\begin{aligned}
D= & D_{0}+D_{1}, \\
D_{0}= & \frac{E_{0} h_{0}^{3}}{12\left(1-v^{2}\right)}\left[4-6 \frac{1-\beta_{1} \beta_{2}^{2}}{1+\beta_{1} \beta_{2}}+3 \frac{\left(1-\beta_{1} \beta_{2}^{2}\right)^{2}}{\left(1+\beta_{1} \beta_{2}\right)^{2}}\right], \\
D_{1}= & \frac{E_{1} h_{0}^{3}}{12\left(1-v^{2}\right)}\left[4 \beta_{2}^{3}+6 \beta_{2}^{2} \frac{1-\beta_{1} \beta_{2}^{2}}{1+\beta_{1} \beta_{2}}\right. \\
& \left.+3 \beta_{2} \frac{\left(1-\beta_{1} \beta_{2}^{2}\right)^{2}}{\left(1+\beta_{1} \beta_{2}\right)^{2}}\right],
\end{aligned}
$$

where $D_{0}$ and $D_{1}$ are the stiffnesses, $h_{0}$ and $h_{1}$ are the thicknesses, and $E_{0}$ and $E_{1}$ are the Young's moduli of the metal and piezoelectric element, respectively, $v$ is Poisson's ratio, $\beta_{1}=E_{1} / E_{0}$, and $\beta_{2}=h_{1} / h_{0}$.

The wave number for the natural angular frequency $\omega_{0}$ and the angular frequency of the exciting force, $\omega$, are defined as

$$
\kappa=\left(\frac{\rho h}{D} \omega^{2}\right)^{\frac{1}{4}} \text { and } \kappa_{0}=\left(\frac{\rho h}{D} \omega_{0}^{2}\right)^{\frac{1}{4}} .
$$

The solution of Eq. (30) is the sum of the general solution of the homogeneous equation and the particular solution. The homogeneous and inhomogeneous equations of Eq. (30) in a steady state are expressed as

$$
\nabla^{4} \phi_{\mathrm{g}}-\kappa_{0}^{4} \phi_{\mathrm{g}}=0
$$

and

$$
\nabla^{4} \phi_{\mathrm{p}}-\kappa^{4} \phi_{\mathrm{p}}=\frac{F}{D r} \delta\left(\boldsymbol{r}-\boldsymbol{r}_{0}\right) .
$$

The general solution of the homogeneous equation (A.4) is represented by using the wave number for the $n$th natural mode $\kappa_{n}$ as 


$$
\begin{aligned}
\phi_{\mathrm{g}}= & \sum_{n=0}^{N}\left[J_{n}\left(\kappa_{n} r\right)\left(A_{n} \cos (n \theta)+B_{n} \sin (n \theta)\right)\right. \\
& \left.+I_{n}\left(\kappa_{n} r\right)\left(C_{n} \cos (n \theta)+D_{n} \sin (n \theta)\right)\right] .
\end{aligned}
$$

By letting the number $n$ be zero for vibration around the fundamental natural frequency, Eq. (31) is obtained. The particular solution of Eq. (A.5) is obtained as Eq. (32) using the wave number $\kappa$.

The ratio of the coefficients $A_{0}$ and $C_{0}$ in Eq. (31) is defined by the boundary condition of the bending plate. The edge of the disk-shaped diaphragm is free; therefore, the general solution of the homogeneous equation (31) is derived by the condition that the resultant share force and bending moment are equivalent to zero at the edge. Accordingly, the boundary conditions for diaphragm 2 can be expressed as

$$
Q_{\mathrm{g} r}=-D \frac{\partial}{\partial r} \nabla^{2} \phi_{\mathrm{g}}=0
$$

and

$$
M_{\mathrm{g} r \theta}=(1-v) D\left(\frac{1}{r} \frac{\partial^{2}}{\partial r \partial \theta}-\frac{1}{r^{2}} \frac{\partial}{\partial \theta}\right) \phi_{\mathrm{g}}=0 .
$$

In addition, the piezoelectric element applies a bending moment to diaphragm 1 , which is expressed as

$$
M^{*}=E_{1} d_{31} V h_{0} \frac{1+\beta_{2}}{2\left(1+\beta_{1} \beta_{2}\right)} .
$$

Thus, the boundary condition of the bending moment for diaphragm 1 is expressed as

$$
M_{\mathrm{g} r \theta}+M^{*}=0 .
$$

Finally, the magnitude of the general solution of the homogeneous equation, $\phi_{\mathrm{g}}$, can be determined with the Rayligh-Ritz method. The potential of the bending disk is expressed in polar coordinates as

$$
\begin{aligned}
U= & \frac{D}{2} \int_{0}^{a} \int_{0}^{2 \pi}\left[\left(\frac{\partial^{2} \phi}{\partial r^{2}}+\frac{1}{r} \frac{\partial \phi}{\partial r}+\frac{1}{r^{2}} \frac{\partial^{2} \phi}{\partial \theta^{2}}\right)^{2}\right. \\
& -2(1-v)\left[\frac{\partial^{2} \phi}{\partial r^{2}}\left(\frac{1}{r} \frac{\partial \phi}{\partial r}+\frac{1}{r^{2}} \frac{\partial^{2} \phi}{\partial \theta^{2}}\right)\right. \\
& \left.\left.-\left(\frac{1}{r^{2}} \frac{\partial \phi}{\partial \theta}-\frac{1}{r} \frac{\partial^{2} \phi}{\partial r \partial \theta}\right)^{2}\right]\right] r d r d \theta .
\end{aligned}
$$

The energy of the exciting force is written as the product of the pressurized area and the sum of the exciting force as

$$
F=f \int_{0}^{\eta} \int_{0}^{2 \pi} \phi r d r d \theta
$$

Here, $\eta$ is the radius of the pressurized area.

The kinetic energy of the bending disk is written as

$$
K=\frac{1}{2} \rho h \int_{0}^{a} \int_{0}^{2 \pi}\left(\frac{d \phi}{d t}\right)^{2} r d r d \theta
$$

The coefficient of the general solution [Eq. (31)] (found by using the Rayligh-Ritz method) is then the solution of the following equation:

$$
\frac{\partial}{\partial A_{0}}(U+F+K)=0 .
$$

